\title{
Correlation of Pyruvate Kinase M2 Expression with Clinicopathological Data in Ovarian Cancer
}

\author{
CHRISTOPHER KOBIERZYCKI ${ }^{1}$, ALEKSANDRA PIOTROWSKA ${ }^{1}$, KRZYSZTOF LATKOWSKI $^{2}$, \\ MACIEJ ZABEL ${ }^{1,3}$, EWA NOWAK-MARKWITZ ${ }^{4}$, MAREK SPACZYNSKI ${ }^{4}$, WITOLD KEDZIA ${ }^{5}$, \\ BARTOSZ PULA $^{1}$, MARZENNA PODHORSKA-OKOLOW ${ }^{1}$ and PIOTR DZIEGIEL ${ }^{1,6}$ \\ Departments of ${ }^{1}$ Human Morphology and Embryology, and ${ }^{2}$ Gynecology and Obstetrics, \\ Wroclaw Medical University, Wroclaw, Poland; \\ Departments of ${ }^{3}$ Histology and Embryology, ${ }^{4}$ Gynecologic Oncology, and \\ ${ }^{5}$ Gynecology, University of Medical Sciences, Poznan, Poland; \\ ${ }^{6}$ Department of Physiotherapy, University School of Physical Education, Wroclaw, Poland
}

\begin{abstract}
Background/Aim: It has been shown in many studies that expression of pyruvate kinase $(P K)$ enzyme plays a key role during cellular metabolism. There is evidence that cancer cells manifesting very dynamic proliferation may control their division in various mechanisms, i.a. by expression of PKM2 isoform. The exact role of PKM2 in ovarian cancer $(O C)$ cells and cancer associated fibroblasts (CAFs) have not been elucidated. Materials and Methods: The present study was focused on analysis of PKM2 expression in cancer cells and CAFs in 97 OC cases, mostly of serous histological type. Moreover, relationships between expression of PKM2 and proliferation (Ki-67; MCM-2, -3, -7; cyclin D1), vascular (CD31, D2-40) and mesenchymal (Vim and $\alpha S M A)$ markers as well as receptors (ER, PR, HER2, EGFR) were examined. All observations were evaluated in regard to available clinicopathological data. Results: The expression of PKM2 was disclosed only in cytoplasm of OC cells. No statistically significant correlation between PKM2 and tested markers was found. In regard to available clinicopathological data only an increasing trend of PKM2 expression with increasing grade of histological malignancy $G$ was found ( $p=0.07)$. Conclusion: Due to achieved results concerning expression of PKM2 there is a lack of evidence for its diagnostic and prognostic usage in $O C$.
\end{abstract}

Correspondence to: Dr. Christopher Kobierzycki, Department of Human Morphology and Embryology, Division of Histology and Embryology, Wroclaw Medical University, Chalubinskiego 6a, 50368 Wroclaw, Poland. Tel: +48 717841682, Fax: +48 717840082, e-mail: ch.kobierzycki@gmail.com

Key Words: Pyruvate kinase, ovarian cancer, immunohistochemistry.
Ovarian cancer (OC) accounts yearly for an estimated 239,000 new cases and 152,000 deaths worldwide, being the seventh most commonly diagnosed cancer among women (1). Lifetime risk of developing OC is $1 / 75$, whereas chance of dying of the disease is $1 / 100$. The overall 5-year relative survival rate varies between $30-40 \%$ (2). There are two hypotheses concerning the exact origin of OC; whether it arises from the ovarian surface epithelium or from the fallopian tube. Neither model has clearly demonstrated superiority over the other (3). OCs also classified into five major histotypes as follows: serous, mucinous, endometrioid, clear cell, and transitional cell (Brenner type), differing in origination, pathogenesis, molecular alterations, risk factors, and prognosis (4). Genetic susceptibility is manifested by rare inherited mutations with high to moderate penetrance. Several reproductive and hormonal factors may decrease risk, including parity, oral contraceptive use, and lactation, while others such as older age at menopause or hormone replacement therapy may increase it. OC risks associated with other gynecological diseases or surgical interventions are less clear. Additional possible risk factors include environmental and lifestyle factors such as asbestos and talc powder exposures, and cigarette smoking $(1,4,5)$. Despite medical, social and economic significance, the etiology of $\mathrm{OC}$ is not completely understood, leading to serious problems with proper diagnostics and finally satisfactory treatment.

Pyruvate kinase (PK) is a ubiquitous enzyme responsible for transfer of a phosphate group from phosphoenolpyruvate to ADP to produce pyruvate and ATP in final rate-limiting step of glycolysis (6). Four isoforms of PK were described, i.e. PKL and PKR expressed in the liver and erythrocytes, respectively, as well as PKM1 and PKM2 expressed in different types of cells and tissues (7). As they are switch-enzymes of glycolysis it was postulated they are responsible for Warburg effect (8). In this phenomenon cancer cells predominantly produce energy 
by a high rate of glycolysis followed by lactic acid fermentation rather than by a comparatively low rate of glycolysis followed by oxidation of pyruvate in mitochondria as in most normal cells. Rapidly dividing tumor cells may demonstrate glycolytic rates up to 200-times higher than in normal tissues cells. This mechanism enables proliferation and invasion even in unfavorable environmental conditions (e.g. anaerobic) that for normal cells is a signal to reduce activity (9). Moreover, PKM2 expression has been found to be increased in many cancer types $(7,10)$, and replacement of PKM2 with PKM1 has been found to inhibit aerobic glycolysis and tumor growth (11). These findings point to a crucial role for expression of PKM2 in tumor growth, whereas results of studies are still not conclusive.

To the Authors' knowledge, there is only one study analyzing expression of PKM2 only in cancer cells of OC. Therefore, the objective of the study was assessment of relationship between PKM2 expression in OC cells and cancer associated fibroblasts (CAFs) as well expression of mentioned markers and clinicopathological data.

\section{Materials and Methods}

Patients. The study was conducted on 97 archival paraffin blocks with OC samples obtained during radical ovariectomy in the Department of Gynecologic Oncology at the University of Medical Sciences in Poznan, Poland. All patients received surgical and chemical treatment according to running protocols. All cases were analyzed by age, histological type, malignancy grade and stage according to the 1988 International Federation of Gynecology and Obstetrics (FIGO) classification. Histological types were determined according to the World Health Organization (WHO) criteria (12). Patient's ages at the time of the diagnosis ranged from 31 to 79 (median 50) years. Based on the literature data, patients were divided into three age groups: $<45$ years old, $45-55$ and $>55$ years old. By histological verification, $79 / 97(81.4 \%)$ cases were confirmed to be serous and 18/97 (18.6\%) endometrioid OC. According to the WHO classification of histological malignancy grade, $17 / 97$ cases were graded as G1 (17.5\%), 38/97 as G2 (39.2\%) and $42 / 97$ as G3 (43.3\%). Based on the FIGO classification, 10/97 cases were evaluated as stage I $(10.3 \%), 12 / 97$ cases as stage II $(12.4 \%), 72 / 97$ cases as stage III $(74.2 \%)$ and $3 / 97$ cases as stage IV $(3.1 \%)$. The mean observation time was 43.2 (range=4-98) months. During this time, 79 of the patients deceased.

Immunohistochemistry (IHC). Primarily, 7- $\mu$ m-thick paraffin sections were stained with haematoxylin and eosin (HE) for diagnosis verification. Subsequently, IHC reactions were performed on $4-\mu \mathrm{m}-$ thick paraffin sections according to a previously established protocol (13). The sections were deparaffinized, antigens retrieved and endogenous peroxidase was blocked using EnVision FLEX Peroxidase-Blocking Reagent (5 min incubation at room temperature, RT). Subsequently, primary antibodies directed against PKM2 (15822-1-AP, 1:200; Proteintech, Manchester, UK), minichromosome maintenance protein 2 (MCM-2, clone CRCT2.1, 1:25; Novocastra, Leica, Nussloch, Germany), cyclin D1 (clone EP12, RTU; Dako, Glostrup, Denmark), estrogen receptor (ER, clone 1D5, RTU; Dako,
Glostrup, Denmark), progesterone receptor (PR, clone Pgr636, RTU; Dako, Glostrup, Denmark), vimentin (Vim, clone V9, RTU; Dako, Glostrup, Denmark), alpha smooth muscle actin ( $\alpha$ SMA, clone 1A4, RTU; Dako, Glostrup, Denmark), human epithelial growth factor receptor 2 (HER-2, 1:200, Dako, Glostrup, Denmark), epithelial growth factor receptor (EGFR, VG1, 1:50 + linker; Dako, Glostrup, Denmark) were applied and incubated at RT for $20 \mathrm{~min}$ in an automated staining platform Link48 Autostainer to ensure repeatable reaction conditions. Then, EnVision FLEX/horseradish peroxidase (HRP) secondary antibodies were applied for $20 \mathrm{~min}$ at RT with following usage of 3,3'-diaminobenzidine tetrachlorohydrate (DAB+chromogen). Finally, the sections were counterstained with Mayer's haematoxylin, dehydrated in alcohol $(70 \%, 96 \%, 99.8 \%)$ and xylene and then mounted using SUB-X Mounting Medium. All reagents and equipment (except separately described antibodies) were obtained from Dako, Glostrup, Denmark.

Evaluation of IHC reactions. IHC reactions were analyzed under a BX-41 light microscope (Olympus, Tokyo, Japan). Cytoplasmic expression of PKM2, Vim and $\alpha$ SMA was evaluated utilizing a 12point semi-quantitative immunoreactive score (IRS), according to Remmele and Stegner (14). The scale is based on the percentage of cells showing positive reaction ( 0 : absence of cells with positive reaction, $1: 1-10 \%$ cells, $2: 11-50 \%, 3: 51-80 \%$, 4 : over $80 \%$ cells with positive reaction), as well as the intensity of the reaction $(0$ : no reaction, 1: low intensity reaction, 2 : moderate intensity reaction, 3: intense reaction). Nuclear expression of MCM-2, Cyclin D1, ER, PR was evaluated utilizing above described percentage scale with quantification labeled nuclei of tumor cells, whereas membranous expression of HER-2 and EGFR was evaluated with usage of described above intensity reaction scale. The IHC reactions in coded preparations were independently evaluated by two pathologists. Moreover, in doubtful cases, re-evaluation with a double-headed microscope was performed until a consensus was achieved.

Statistical analysis. For assessment of relationships between results of performed reactions (PKM2, MCM-2, cyclin D1, Vim, $\alpha$ SMA, ER, PR, HER2, EGFR) as well already available from other studies (i.e. Ki-67; MCM-3, -7, CD31, D2-40) and clinicopathological data Prism 5.0 (GraphPad, La Jolla, CA, USA) statistical software was used. Relationships between the expression of the studied antigens and patients clinicopathological parameters were analyzed using Kruskal-Wallis, Mann-Whitney $U$-test, Fisher's exact and Spearman's correlation tests. Kaplan-Meier method and the log-rank test was used to analyze patient survival. The hazard ratio (HR) and 95\% confidence interval (CI) were estimated for each variable. In all analyses, results were considered to be statistically significant when $p$ was less than 0.05 .

\section{Results}

The expression of PKM2 was disclosed only in cytoplasm of OC cells (Figure 1A-C). Expression of ER, PR, MCM-2, cyclin D1 in nuclei whereas HER-2 and EGFR in cell membrane of OC cells (Figure 2A-F). Expression of Vim and $\alpha \mathrm{SMA}$ was observed in the cytoplasm of CAFs (Figure 2G-H).

To verify potential utilization of PKM2 as a proliferation marker its expression was correlated with Ki-67, cyclin D1, MCM-2, -3 and -7. Unfortunately, Spearman rank test 

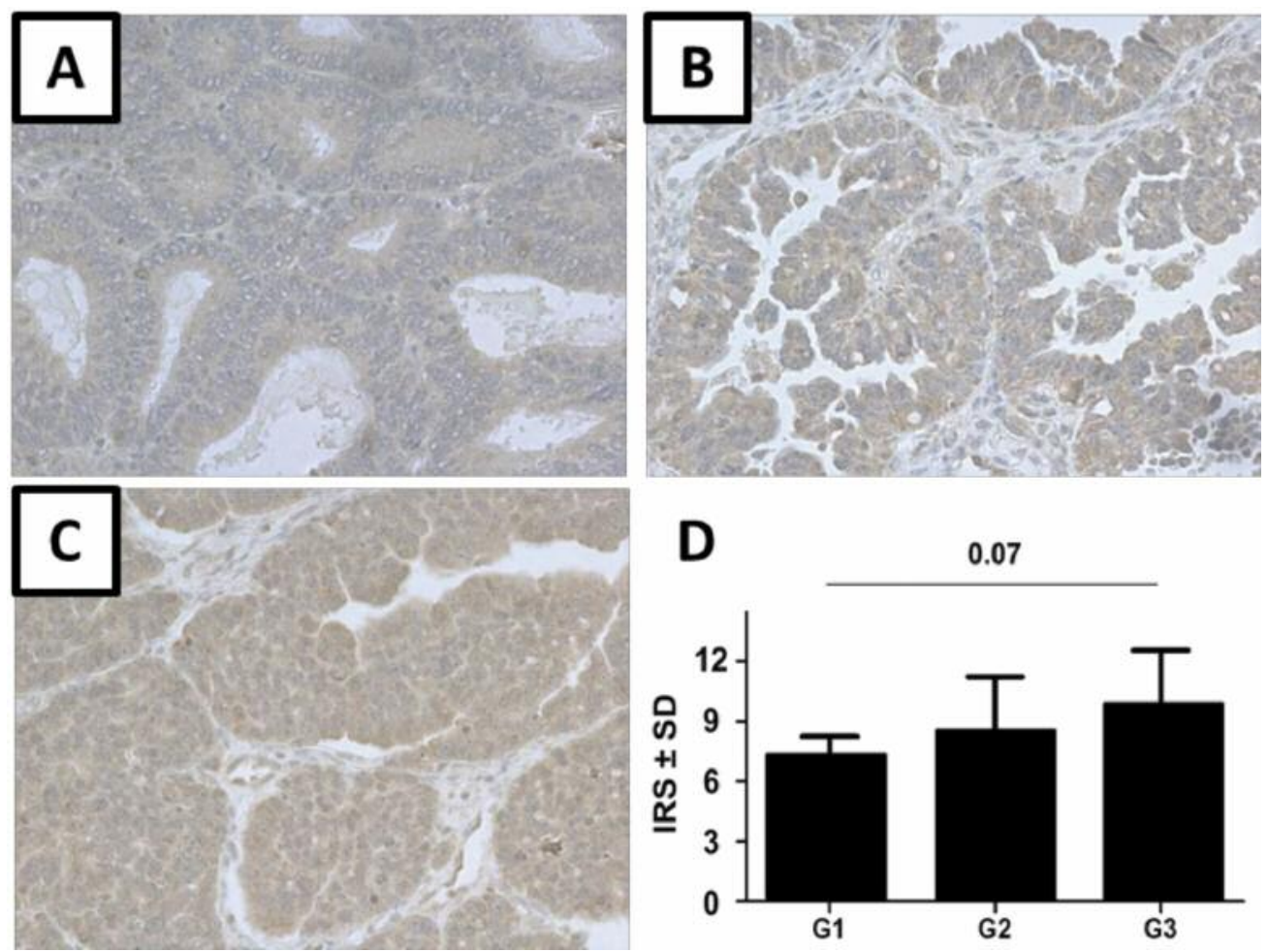

Figure 1. Immunohistochemical expression of pyruvate kinase M2 in ovarian cancer cells. Cytoplasmic reaction in different grades of histological malignancy: $G 1$ (A), G2 (B), G3 (C). Magnification $\times 200$. The graph shows analysis of immunoreactive score (IRS) by the Kruskal-Wallis test (D).

Table I. Spearman correlation test results. Statistically significant associations given in bold.

\begin{tabular}{|c|c|c|c|c|c|c|}
\hline & PKM2 & $\mathrm{Ki}-67$ & Cyclin D1 & MCM-2 & MCM-3 & MCM-7 \\
\hline PKM2 & ---- & $\mathrm{r}=0.177 p=0.097$ & $\mathrm{r}=0.344 p=0.237$ & $\mathrm{r}=0.455 p=0.145$ & $\mathrm{r}=0.419 p=0.196$ & $\mathrm{r}=0.322 p=0.102$ \\
\hline Ki-67 & & ---- & $r=0.724 p<0.05$ & $\mathrm{r}=0.532 p<0.01$ & $\mathrm{r}=0.483 p<0.001$ & $r=0.622 p<0.001$ \\
\hline Cyclin D1 & & & ---- & $r=0.263 p<0.05$ & $\mathrm{r}=0.219 p=0.089$ & $\mathrm{r}=0.312 p<0.01$ \\
\hline MCM-2 & & & & ---- & $\mathrm{r}=0.711 p<0.01$ & $\mathrm{r}=0.577 p<0.001$ \\
\hline MCM-3 & & & & & ---- & $\mathrm{r}=0.503 p<0.001$ \\
\hline MCM-7 & & & & & & ---- \\
\hline
\end{tabular}

disclosed insignificant relationships. However, between other markers, especially MCM family proteins even stronger correlations were found (Table I). In the next step associations between PKM2 and receptors expressions were tested. Due to presence of convincing expression of HER2 and EGFR only in 4 and 17 cases, respectively, they were not included into further analysis. Only ER and PR expression correlated strongly $(\mathrm{r}=0.723, p<0.001)$.

In the tumor stroma expression of PKM2 in CAFs was not disclosed. The expression of Vim and $\alpha$ SMA correlated strongly $(\mathrm{r}=0.893, p<0.001)$, while statistically significant associations with D2-40 and CD31 were not observed.
In regard to available clinicopathological data only an increasing trend of PKM2 expression with increasing grade of histological malignancy $\mathrm{G}$ was found ( $p=0.07$, KruskalWallis test; Figure 1D). Moreover, univariate survival analysis revealed no prognostic significance.

\section{Discussion}

PKM2 is instrumental to cancer metabolism and hence overexpressed in cancer cells leading to detectable plasma concentrations. It was found to be elevated in many types of cancers, including breast, lung, ovarian, thyroid, prostatic, 

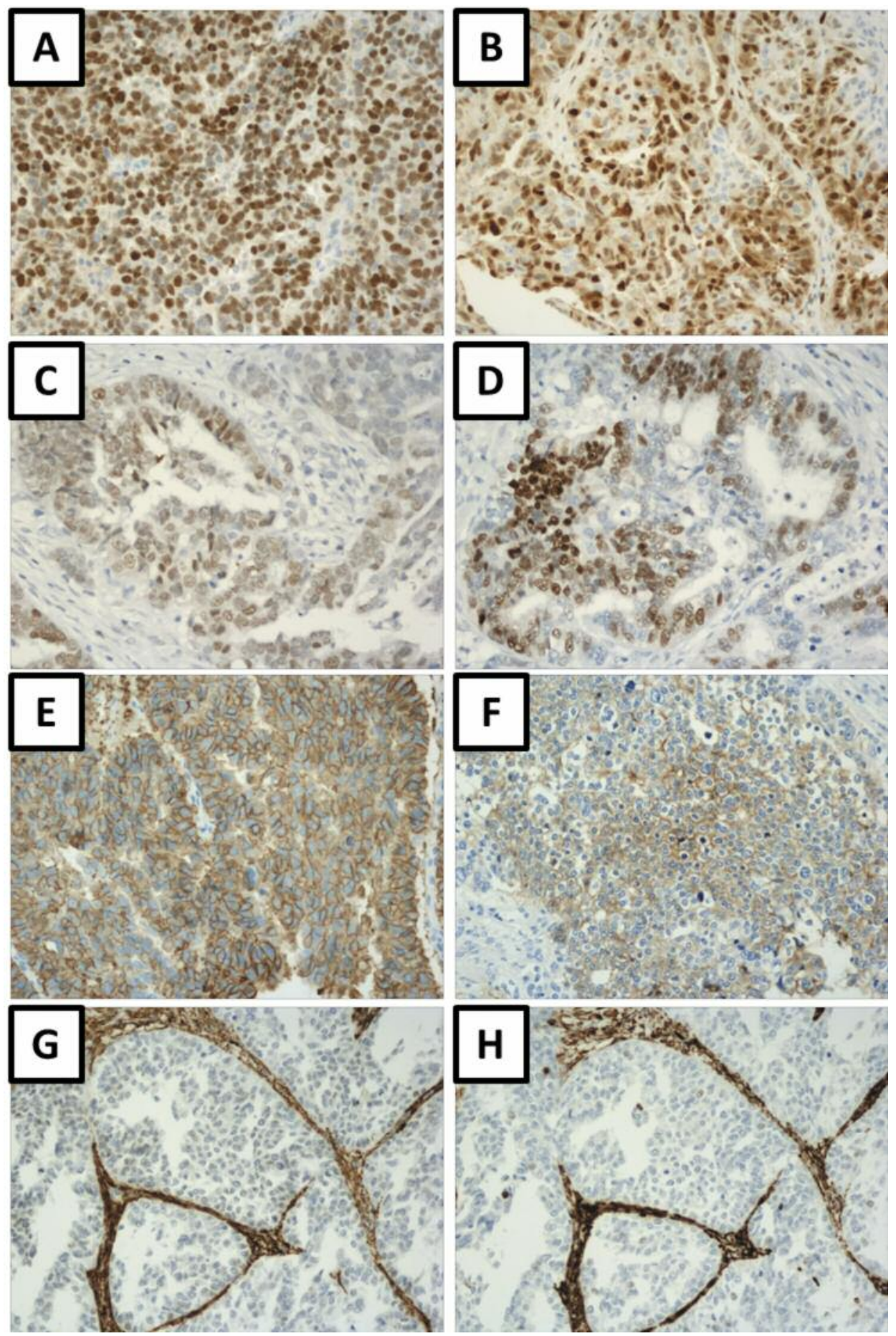

Figure 2. Immunohistochemical expression of minichromosome maintenance protein $2(A)$, cyclin D1 (B), estrogen $(C)$ and progesterone $(D)$ receptors, human epidermal growth factor receptor $2(E)$, epidermal growth factor receptor $(F)$, vimentin $(G)$ and $\alpha$ smooth muscle actin $(H)$. Magnification $\times 200$.

pharyngeal and testicular tumors (15). Moreover, in OC patients its significant raise was shown, particularly in those with higher stage disease. However, potential clinical application needs further evaluation (16). Due to the complex character of the interactions in neoplastic lesions, studies should be separately performed for cancer cells and cancer stroma, mostly CAFs. 
A study by Li et al. was focused on analysis of the role of nitric oxide (iNOS) on OC metabolism, by glycolysis regulation. Authors stated that iNOS has a positive effect on glycolysis by inducing PKM2 nuclear translocation in an EGFR/ERK2 signaling-dependent manner. In response to EGFR activation, which occurs in many types of human cancers, $P K M$ promoter is activated what enhances PKM2 expression, resulting in the Warburg effect and tumorigenesis (17). Moreover, iNOS is overexpressed in OC and correlates with PKM2 expression. It is associated with aggressive phenotype and poor survival outcome in OC patients (18). There is also evidence of shifting PKM2 expression between cytoplasm and nucleus. Due to the fact that PKM2 is a critical rate-limiting enzyme in glycolysis it is highly expressed in the cytoplasm of OC cells (19). Whereas other studies have shown nuclear PKM2 expression, which mediates cell proliferation and metabolic reprogramming in cancer cells (20). This may be a reason of lack of statistically significant correlations between PKM2 (cytoplasmic expression) and proliferation markers. Moreover, MCM family proteins, Ki67 and cyclin D1 correlated with each other well, in concordance with previous studies. Kobierzycki et al. (21) disclosed strong correlation between MCM-3, MCM-7 and Ki-67, whereas Gakiopoulou et al. (22) and Levidou et al. (23) revealed strong positive correlations between MCM-2, MCM-5 and Ki-67 antigen in OC samples. In perspective of histogenesis of OC as well as known metabolic pathways we decided to evaluate the expression of HER-2 and EGFR, however due to a small sample size it was not statistically analyzed. Steroid hormones correlated strongly ( $\mathrm{r}=0.723$, $p<0.001$ ), whereas possibly due to the fact that nuclear localization of PKM2 controls proliferation processes, there was no association between ER, PR and PKM2.

There is a strong rationale for separate analysis of cancers cells and stroma, due to evident strong cross-talk between these two compartments. It was shown that fibroblasts expressing PKM1 or PKM2 greatly promoted the growth of co-injected MDA-MB-231 breast cancer cells, without an increase in tumor angiogenesis (24). There are no studies performed on OC samples verifying PKM2 expression in CAFs. In our study we did not disclose expression in CAFs and subsequently we could not examine association with potential angiogenesis. Whereas we performed IHC reactions against Vim and $\alpha$ SMA receiving clear reaction and strong correlation between these markers $(\mathrm{r}=0.893, p<0.001)$. Probably, it would be beneficial is such situation to conduct further studies by Western-blotting and laser-microdissection of stromal cells.

Additionally, in some cancers PKM2 may have prognostic and predictive value. It has been linked to poor survival in signet ring cell cancer and to advanced stage in colorectal cancer (25). However, the results by Hjerpe et al. (26) indicate that PKM2 might not provide prognostic information in OC, similarly to our present observation.
Whereas, PKM2 has been implicated in platinum-resistance in OC cell lines. In performed experiments, silencing of PKM-2 and MDR-1 sensitized multidrug-resistant OC cells to paclitaxel in a murine model of $\mathrm{OC}$, revealing potential predictive value of PKM2 $(27,28)$.

Due to achieved results concerning expression of PKM2 there is a lack of evidence for its diagnostic and prognostic usage in OC. However, in perspective of existing evidence of PKM2 expression in different types of tumors as well as the presented complex pattern of expression, i.e. cytoplasmic and nuclear, further studies seem to be necessary.

\section{Acknowledgements}

This study was supported by a grant of the National Science Centre decision no. DEC-2012/05/N/NZ4/02537.

\section{References}

1 Reid BM, Permuth JB and Sellers TA: Epidemiology of ovarian cancer: a review. Cancer Biol Med 14: 9-32, 2017.

2 Allemani C, Weir HK, Carreira H, Harewood R, Spika D, Wang XS, Bannon F, Ahn JV, Johnson CJ, Bonaventure A, MarcosGragera R, Stiller C, Azevedo e Silva G, Chen WQ, Ogunbiyi OJ, Rachet B, Soeberg MJ, You H, Matsuda T, Bielska-Lasota M, Storm H, Tucker TC and Coleman MP: Global surveillance of cancer survival 1995-2009: analysis of individual data for 25676887 patients from 279 population-based registries in 67 countries (CONCORD-2). Lancet 385: 977-1010, 2015.

3 Klotz DM and Wimberger P: Cells of origin of ovarian cancer: ovarian surface epithelium or fallopian tube? Arch Gynecol Obstet 296: 1055-1062, 2017.

4 McCluggage WG: Morphological subtypes of ovarian carcinoma: a review with emphasis on new developments and pathogenesis. Pathology 43: 420-432, 2011.

5 Prat J: Ovarian carcinomas: five distinct diseases with different origins, genetic alterations, and clinicopathological features. Virchows Arch 460: 237-249, 2012.

6 Yang W and Lu Z: Pyruvate kinase M2 at a glance. J Cell Sci 128: 1655-1660, 2015.

7 Bluemlein K, Grüning NM, Feichtinger RG, Lehrach H, Kofler $\mathrm{B}$ and Ralser M: No evidence for a shift in pyruvate kinase PKM1 to PKM2 expression during tumorigenesis. Oncotarget 2: 393-400, 2011.

8 Warburg O: On the origin of cancer cells. Science 123: 309-314, 1956.

9 Alfarouk KO: Tumor metabolism, cancer cell transporters, and microenvironmental resistance. J Enzyme Inhib Med Chem 31: 859-866, 2016

10 Desai S, Ding M, Wang B, Lu Z, Zhao Q, Shaw K, Yung WK, Weinstein JN, Tan M and Yao J: Tissue-specific isoform switch and DNA hypomethylation of the pyruvate kinase PKM gene in human cancers. Oncotarget 5: 8202-8210, 2014.

11 Christofk HR, Vander Heiden MG, Harris MH, Ramanathan A, Gerszten RE, Wei R, Fleming MD, Schreiber SL and Cantley LC: The M2 splice isoform of pyruvate kinase is important for cancer metabolism and tumour growth. Nature 452: 230-233, 2008 . 
12 Tavassoli FA and Devilee P: Pathology and Genetics of Tumours of the Breast and Female Genital Organs. World Health Organization Classification of Tumours. IARC Press, Lyon, 2003.

13 Kobierzycki C, Pula B, Werynska B, Piotrowska A, Muszczynska-Bernhard B, Dziegiel P and Rakus D: The lack of evidence for correlation of pyruvate kinase M2 expression with tumor grade in non-small cell lung cancer. Anticancer Res 34: 3811-3817, 2014.

14 Remmele W and Stegner HE: Recommendation for uniform definition of an immunoreactive score (IRS) for immunohistochemical estrogen receptor detection (ER-ICA) in breast cancertissue. Pathologe 8: 138-140, 1987.

15 Muñoz-Colmenero A, Fernández-Suárez A, Fatela-Cantillo D, Ocaña-Pérez E, Domínguez-Jiménez JL and Díaz-Iglesias JM: Plasma tumor M2-pyruvate kinase levels in different cancer types. Anticancer Res 35: 4271-4276, 2015.

16 Dew T, Lawton FG, Papadopoulos AJ, Devaja O, Raju KS and Sherwood RA: Tumour M2-PK as a predictor of surgical outcome in ovarian cancer, a prospective cohort study. Eur J Gynaecol Oncol 28: 103-108, 2007.

17 Li L, Zhu L, Hao B, Gao W, Wang Q, Li K, Wang M, Huang M, Liu Z, Yang Q, Li X, Zhong Z, Huang W, Xiao G, Xu Y, Yao K and Liu Q: iNOS-derived nitric oxide promotes glycolysis by inducing pyruvate kinase M2 nuclear translocation in ovarian cancer. Oncotarget 8: 33047-33063, 2017.

18 Wang X, Duan W, Li X, Liu J, Li D, Ye L, Qian L, Yang A, Xu Q, Liu H, Fu Q, Wu E, Ma Q and Shen X: PTTG regulates the metabolic switch of ovarian cancer cells via the c-myc pathway. Oncotarget 6: 40959-40969, 2015.

19 Yang W, Xia Y, Cao Y, Zheng Y, Bu W, Zhang L, You MJ, Koh MY, Cote G, Aldape K, Li Y, Verma IM, Chiao PJ and Lu Z: EGFR-induced and PKC $\varepsilon$ monoubiquitylation-dependent NF- $x \mathrm{~B}$ activation upregulates PKM2 expression and promotes tumorigenesis. Mol Cell 48: 771-784, 2012.

20 Yang W, Zheng Y, Xia Y, Ji H, Chen X, Guo F, Lyssiotis CA, Aldape K, Cantley LC and Lu Z: ERK1/2-dependent phosphorylation and nuclear translocation of PKM2 promotes the Warburg effect. Nat Cell Biol 14: 1295-1304, 2012.

21 Kobierzycki C, Pula B, Skiba M, Jablonska K, Latkowski K, Zabel M, Nowak-Markwitz E, Spaczynski M, Kedzia W, Podhorska-Okolow $\mathrm{M}$ and Dziegiel $\mathrm{P}$ : Comparison of minichromosome maintenance proteins (MCM-3, MCM-7) and metallothioneins (MT-I/II, MT-III) expression in relation to clinicopathological data in ovarian cancer. Anticancer Res 33: 5375-5383, 2013
22 Gakiopoulou H, Korkolopoulou P, Levidou G, Thymara I, Saetta A, Piperi C, Givalos N, Vassilopoulos I, Ventouri K, Tsenga A, Bamias A, Dimopoulos MA, Agapitos E and Patsouris E: Minichromosome maintenance proteins 2 and 5 in non-benign epithelial ovarian tumours: Relationship with cell cycle regulators and prognostic implications. Br J Cancer 97: 11241134,2007

23 Levidou G, Ventouri K, Nonni A, Gakiopoulou H, Bamias A, Sotiropoulou M, Papaspirou I, Dimopoulos MA, Patsouris E and Korkolopoulou P: Replication protein A in nonearly ovarian adenocarcinomas: Correlation with MCM-2, MCM-5, Ki-67 index and prognostic significance. Int J Gynecol Pathol 31: 319327, 2012.

24 Chiavarina B, Whitaker-Menezes D, Martinez-Outschoorn UE, Witkiewicz AK, Birbe R, Howell A, Pestell RG, Smith J, Daniel R, Sotgia F and Lisanti MP: Pyruvate kinase expression (PKM1 and PKM2) in cancer-associated fibroblasts drives stromal nutrient production and tumor growth. Cancer Biol Ther 12: 1101-1113, 2011

25 Lim JY, Yoon SO, Seol SY, Hong SW, Kim JW, Choi SH and Cho JY: Overexpression of the M2 isoform of pyruvate kinase is an adverse prognostic factor for signet ring cell gastric cancer. World J Gastroenterol 18: 4037-4043, 2012.

26 Hjerpe E, Brage SE, Carlson J, Stolt MF, Schedvins K, Johansson $\mathrm{H}$, Shoshan $\mathrm{M}$ and Åvall-Lundqvist E: Metabolic markers GAPDH, PKM2, ATP5B and BEC-index in advanced serous ovarian cancer. BMC Clin Pathol 13: 30, 2013.

27 Li SL, Ye F, Cai WJ, Hu HD, Hu P, Ren H, Zhu FF and Zhang DZ: Quantitative proteome analysis of multidrug resistance in human ovarian cancer cell line. J Cell Biochem 109: 625-633, 2010.

28 Talekar M, Ouyang Q, Goldberg MS and Amiji MM: Cosilencing of PKM-2 and MDR-1 sensitizes multidrug-resistant ovarian cancer cells to paclitaxel in a murine model of ovarian cancer. Mol Cancer Ther 14: 1521-1531, 2015.

Received November 13, 2017

Revised November 25, 2017

Accepted November 27, 2017 